\title{
E2F1, un oncogène... suppresseur de tumeur
}

Les protéines E2F sont bien connues comme étant la cible principale des protéines de la famille Rb (produit du gène de susceptibilité au rétinoblastome), que l'on appelle aujourd'hui les "protéines à poche " (pocket proteins). Rb non phosphorylée est liée à des protéines E2F dont elle inhibe la fonction d'activation de gènes nécessaires à la progression dans le cycle cellulaire. Lorsque Rb est phosphorylée en fin de phase Gl, ou bien lorsqu'elle est complexée au produit d'oncogènes viraux de type ElA d'adénovirus, E7 de papillomavirus et antigène $\mathrm{T}$ du virus SV40, les facteurs E2F sont libérés et jouent leur rôle d'activateur transcriptionnel $\left(m / s n^{\circ} 4\right.$, vol. 5, p. 259). Par conséquent, ces facteurs E2F sont généralement considérés comme des oncogènes normalement inactivés par les anti-oncogènes de la famille Rb. On connaît aujourd'hui cinq membres de cette famille E2F; E2F , $\mathrm{E} 2 \mathrm{~F} 2$ et E2F3 peuvent se fixer à $\mathrm{Rb}$ alors que E2F4 et E2F5 se fixent aux protéines à poche pl07 et p130. Le facteur E2Fl a été le mieux caractérisé. Son hyperexpression dans les cellules en culture provoque, soit leur prolifération, soit, dans certaines conditions, leur apoptose. De plus, on sait que le complexe $\mathrm{E} 2 \mathrm{Fl} / \mathrm{Rb}$ peut se fixer aux cibles d'ADN de E2F et réprimer activement la transcription de certains gènes impliqués dans la progression dans le cycle cellulaire $\left(m / s n^{\circ} 9\right.$, vol. 11, p. 1358). Par conséquent, le phénotype d'une carence en facteur E2Fl n'était pas facilement prévisible. On pouvait s'attendre à une absence totale de phénotype du fait de la redondance fonctionnelle entre les différentes protéines E2F; ou bien à une absence de développement normal, du fait du rôle de E2Fl dans la prolifération cellulaire; ou bien à une diminution de l'apoptose; ou enfin à une sensibilité aux tumeurs par perte de certains des effets de Rb sur l'inhibition spécifique de gènes inducteurs de prolifération. Deux équipes américaines de Nouvelle-Angleterre (Massachusetts) publient l'étude phénotypique de souris dont les deux allèles du gène $E 2 F 1$ ont été invalidés par recombinaison homologue. Field et al. ont réalisé une délétion du quatrième exon du gène [1] alors que Yamasaki el al. ont obtenu une délétion des exons 2 et 3 [2]. Les phénotypes décrits comportent des points communs et quelques différences. Pour les deux équipes, le déficit en E2F n'empêche pas un développement normal et les animaux sont viables et fertiles. Field $e t$ al. décrivent deux types d'anomalies: un excès de lymphocytes $T$ mûrs chez les animaux jeunes et une prolifération thymique intéressant probablement des thymocytes du cortex chez les animaux âgés. Ces auteurs notent également une discrète atrophie testiculaire [1] qui semble plus importante chez les animaux décrits par Yamasaki el al. [2]. Ces derniers auteurs notent une dysplasie diffuse des glandes exocrines (glandes salivaires, pancréas exocrine) et, chez l'animal âgé de plus d'un an, le développement de nombreux types de tumeurs parmi lesquelles dominent les cancers du tractus urogénital, les tumeurs du poumon et les lymphomes [2]. Ainsi, le déficit en E2Fl n'empêche pas un développement embryonnaire strictement normal, preuve que la fonction de E2Fl sur la progression du cycle cellulaire est partiellement redondante, probablement avec celle des autres protéines
E2F. La symptomatologie dominante de ce déficit semble être en rapport avec le rôle de E2Fl dans l'induction de l'apoptose et dans la coopération avec la protéine $\mathrm{Rb}$, produit d'un anti-oncogène: les souris déficientes en E2F1 sont susceptibles au développement tumoral, et cette susceptibilité se manifeste même chez les hétérozygotes [2]. Cependant, le phénotype dû au déficit en E2Fl est bien moins sévère que celui du déficit en $\mathrm{Rb}$ qui entraîne une mortalité in utero. C'est que les fonctions de $\mathrm{Rb}$ ne passent évidemment pas toutes par la liaison à E2F.

Quant aux différences entre les phénotypes observés par les deux équipes, elles pourraient être liées à ce que les événements de recombinaison homologue ne sont pas identiques et que les gènes modifiés aboutissent néanmoins à un certain niveau de synthèse de protéines tronquées qui pourraient interférer de manière différente avec les systèmes de contrôle de la prolifération cellulaire. Malgré ces différences, il apparaît bien que $\mathrm{E} 2 \mathrm{~F}$ peut être considéré comme un oncogène à fonction redondante... et comme un gène suppresseur de tumeur dont toutes les fonctions ne peuvent être remplacées par les autres protéines E2F.

A.K.

1. Field SJ, Tsai F-Y, Kuo F, Zubiaga AM, Kaelin WG, Livingston DM, Orkin SH, (rreenberg ME. E2F-l functions in mice to promote apoptosis and suppress proliferation. Cell 1996; 85: 549-61.

2. Yamasaki L., Jacks T, Bronson R, Goillot E, Harlow F, Dyson NJ. Tumor induction and tissue atrophy in mice lacking E2F-1. Cell 1996; 85: 537-48. 